(C) 2017 IEEE. Personal use of this material is permitted. Permission from IEEE must be obtained for all other uses, in any current or future media, including reprinting/republishing this material for advertising or promotional purposes, creating new collective works, for resale or redistribution to servers or lists, or reuse of any copyrighted component of this work in other works. 


\title{
Analytical Modeling of Manufacturing Imperfections in Double Rotor Axial Flux PM Machines: Effects on Back EMF
}

\author{
Baocheng Guo ${ }^{1}$, Yunkai Huang ${ }^{1}$, Fei Peng ${ }^{1}$, Youguang Guo ${ }^{2}$ and Jianguo $\mathrm{Zhu}^{2}$ \\ ${ }^{1}$ School of Electrical Engineering, Southeast University, Nanjing 210096, China \\ ${ }^{2}$ School of Electrical, Mechanical and Mechatronic Systems, University of Technology Sydney, Sydney, Australia
}

\begin{abstract}
In this paper, a general analytical model is proposed to calculate the back electromotive forces (EMFs) of various manufacturing imperfections in the double rotor axial flux permanent magnet (DRAFPM) machine. Firstly, the axial and circumferential flux density in the air gap under healthy condition is developed by Maxwell's equations and Schwarz-Christoffel mapping. Secondly, the equations of air gap length and radii are introduced to calculate the flux density with manufacturing imperfections. The back EMFs of each case are calculated. For each case, FEM models are developed to validate the analytical model, and the results show that the analytical model predictions match well with the FE results. Finally, the analytical model is verified by experimental results.
\end{abstract}

Index Terms-Analytical model, double rotor AFPMMs, Manufacturing imperfections, Back EMFs.

\section{INTRODUCTION}

$\mathrm{T}_{\mathrm{t}}$ AKING BENEFIT FROM the compact mechanical structure, the axial flux permanent magnet machines (AFPMMs) are of a high power density. Therefore, they are widely used in electrical vehicles, hybrid electric vehicles (HEV) and flywheel energy storage systems (FESS). However, the small contact surface between rotor plate and shaft, which makes the rotor predisposed to eccentricity, is a problem and must be considered in assembling progress. Various types of manufacturing imperfections could be found in [1], and they cause serious problems such as unbalanced magnetic force and mechanical vibration.

Several techniques are adopted to analyze imperfect conditions, e.g., analytical method, finite element (FE) method [2] and hybrid method [3]. AFPMMs has strong 3D effects. A full time stepping 3D FE model can be used to obtain accurate results. However, 3D FE model is very time consuming. Although multi-slice 2D FE model has less computational time, it still costs too much time on modeling different geometries. Another alternative is analytical method. It is faster than FE method and can obtain acceptable results. therefore, analytical method is widely adopted by electric machine (EM) designers.

From literature survey, subdomain (SD) method is more accuracy than other analytical approaches, because it can predict accurate cogging torque. However, since several parameters are changed, it is not suitable to deal with EM with imperfections. In this paper, based on quasi 3D-2D method, Fourier series method combined with Schwarz-Christoffel

Manuscript received April 1, 2015; revised May 15, 2015 and June 1, 2015; accepted July 1, 2015. Date of publication July 10, 2015; date of current version July 31, 2015.

This work was supported in part by the National Nature Science Foundation of China (Project Number 51377019), the Scientific and Technological Innovation Program of Jiangsu Province (Project Number BY2014127-15), and the Qing Lan Project.
(SC) mapping are used to calculate the magnetic flux density in the air gap under both healthy condition and manufacturing imperfections.

This paper is organized as follows. The prototype machine and analytical model are introduced in section II and III. The types of eccentricities are discussed in section IV and the back EMFs is then discussed in section V. The experiment results are presented in section VI. Finally, conclusion is draw in section VII.

\section{DESCRIPTION OF PROTOTYPE MACHINE}

Figure 1 (a) shows the construction of the studied machine, Fig. 1 (b) and (c) show the principle of quasi 3D-2D method used in this paper. The SMC material is used as stator core to reduce iron loss [4]. Dimensions and specifications of the investigated machine can be seen in [5].

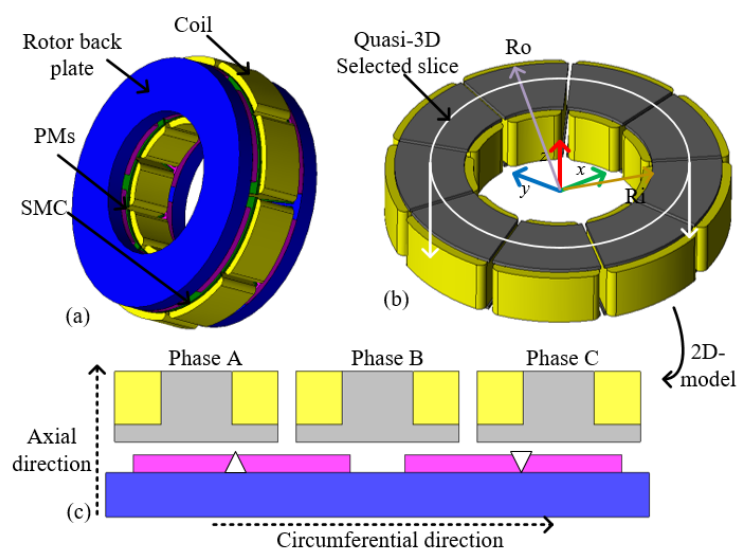

Fig. 1. (a) Construction of the investigated AFPMM. (b) and (c) The principle of quasi 3D-2D method.

\section{DESCRIPTION OF ANALYTICAL MODEL}

In this section, the approach in [6] is used to calculate the magnetic induction in the air gap caused by the magnets. The following assumptions are made to reduce the complexity of the computations.

1) The magnetic material is uniformly magnetized and the relative recoil permeability $\mu_{r}$ is constant and has a value close 
to unity.

2) Magnetic saturation is ignored and the rotor iron cores have infinite magnetic permeability.

3) Eddy current effects are neglected, which avoids the need of the complex eddy current field formulation.

A 2D model developed in the 2D Cartesian coordinate is shown in Fig.1 (c), the average radius of a particular $i$-th layer $\left(R_{\text {ave }}^{i}\right)$ is

$$
\begin{gathered}
R_{\text {ave }}^{i}=R_{i}+\frac{R_{o}-R_{i}}{2 n_{s}}(2 i-1), \quad i=1,2, \ldots, n . \\
t_{c p}=\frac{R_{o}-R_{i}}{n_{s}}
\end{gathered}
$$

where $R_{o}$ is the outer radius, $R_{i}$ is the inner radius, $n_{s}$ is the number of slices, $t_{c p}$ is the width of each slice.

\section{A. Model of PMs}

Firstly, the magnetic flux distributions under region I and region II are calculated as shown in Fig. 2, assuming that the stator is slotless. The magnetization vector is assumed to be along with the axial direction and is described by a Fourier series as:

$$
M_{n}=\frac{4 B_{r}}{n \pi \mu_{0}} \sin \left(\frac{n \pi \alpha_{p}}{2}\right)
$$

where $n$ is harmonic order, $B_{r}$ is the remnant magnet flux density of PM, $\mu_{0}$ is the permeability of free space, $\alpha_{p}$ is the ratio of magnet pole arc to pole pitch $\left(\tau_{p}\right)$.

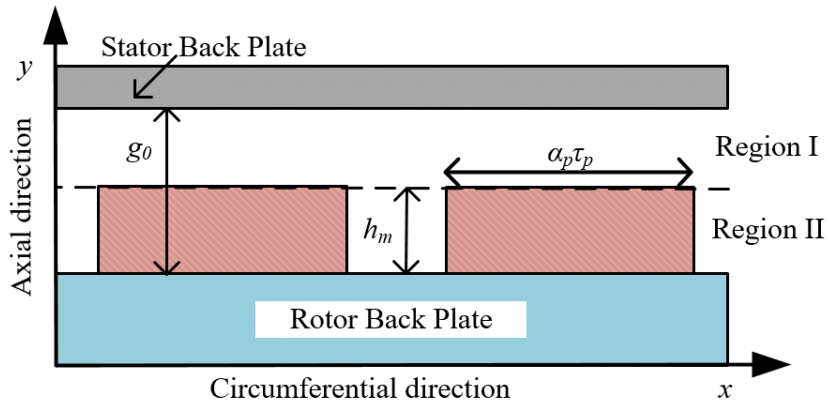

Fig. 2. Model of air gap flux caused by magnets.

This result in two field components in region I (the air space) is:

$$
\begin{aligned}
B_{x I}= & \mu_{0} H_{x I}=\mu_{0} \sum_{n=1,3,5, \ldots}^{\infty} M_{n} \sinh \left(\left(n \pi h_{m} / \tau_{p}\right) / \Delta\right) \\
& \times \sinh \left\{n \pi\left(g_{0}-y\right) / \tau_{p}\right\} \sin \left(n \pi x / \tau_{p}\right) \\
B_{y I}= & \mu_{0} H_{y I}=\mu_{0} \sum_{n=1,3,5, \ldots}^{\infty} M_{n} \sinh \left(\left(n \pi h_{m} / \tau_{p}\right) / \Delta\right) \\
& \times \cosh \left\{n \pi\left(g_{0}-y\right) / \tau_{p}\right\} \cos \left(n \pi x / \tau_{p}\right)
\end{aligned}
$$

where $\tau_{p}$ is pole pitch in circumferential direction, $h_{m}$ is axial thickness of magnets, $g_{0}$ is the axial distance between rotor and stator back plates, and:

$$
\begin{aligned}
\Delta= & \mu_{r} \cosh \left(n \pi h_{m} / \tau_{p}\right) \sin \left\{n \pi\left(g_{0}-h_{m}\right) / \tau_{p}\right\} \\
& +\cos \left\{n \pi\left(g_{0}-h_{m}\right) / \tau_{p}\right\} \sinh \left(n \pi h_{m} / \tau_{p}\right)
\end{aligned}
$$

\section{B. Model of Schwarz-Christoffel Mapping}

Secondly, the effect of stator slotting is included using numerical SC mapping technique described in [7], the complex relative airgap permeance $\left(\lambda^{*}\right)$ is introduced to obtain the final magnet flux in the slot region [8]:

$$
B_{z}=B_{t} \cdot \lambda^{*}=\left(B_{y}+j \cdot B_{x}\right) \cdot\left(\operatorname{Re}\left(\lambda^{*}\right)+j \cdot \operatorname{Im}\left(\lambda^{*}\right)\right)
$$

where $B_{z}$ and $B_{t}$ are the flux densities at $\mathrm{Z}$ and $\mathrm{T}$ domain, as shown in Fig.3.

For AFPMMs, the complex permeance can be calculated as:

$$
\lambda^{*}=\frac{\partial K}{\partial T} \cdot \frac{\partial T}{\partial Z} \cdot \frac{1}{\lambda_{0}}
$$

The transformation is done in two steps, as shown in Fig. 3. the complex plane (Z-plane) is first transformed to the rectangle domain (T-plane), which is then transformed to an annular domain (K-plane). More detailed information could be found in [9].

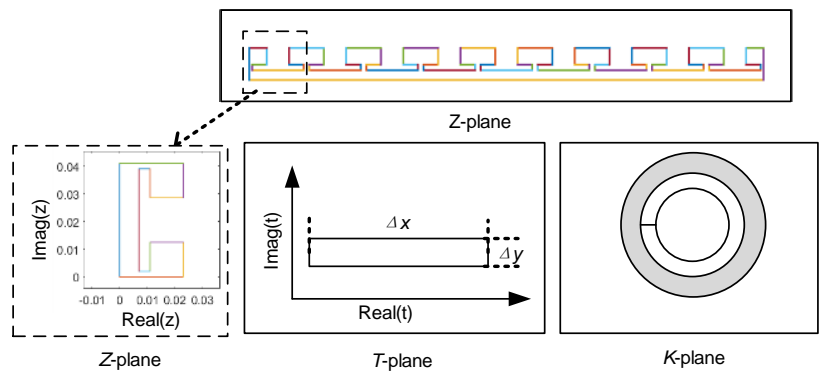

Fig. 3. SC mapping on different planes.

\section{DESCRIPTION OF ECCENTRICITIES}

Static/dynamic axis and angular misalignment eccentricities are considered in this section.

\section{A. Model of Angular Misalignment}

The angular misalignment is caused by the slant rotor plate. The static and dynamic angular misalignment is shown in Fig. 4.

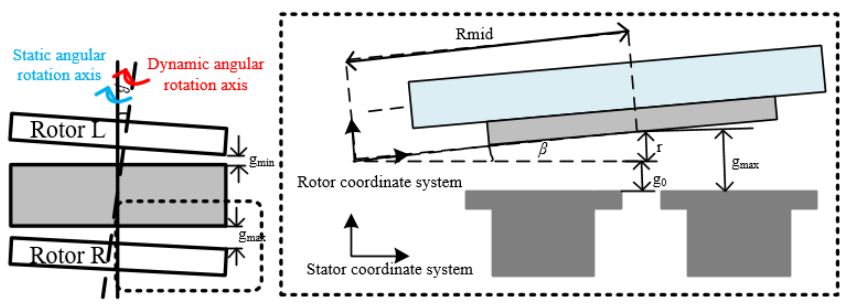

Fig. 4. Configuration of static/dynamic angular misalignment.

The static eccentricity factor (SEF) is used to describe the degree of angular misalignment [10]:

$$
S E F=\frac{|r|}{g_{0}} \times 100 \%
$$

where $r$ is the distance from the normal air gap length $g_{0}$. The airgap length at radius $R$ in static reference frame is deduced as:

$$
g(R, \varphi)=g_{0}\left(1-\frac{R}{R_{\text {mid }}} S E F \cdot \cos \left(\varphi-\gamma_{0}\right)\right)
$$


where $R_{\text {mid }}$ is the average radius and $\phi$ is the angle from a reference point $\left(\gamma_{0}\right)$ of the minimal air gap.

The airgap length of dynamic angular misalignment in static reference frame is:

$$
g(R, \varphi)=g_{0}\left(1-\frac{R}{R_{\text {mid }}} S E F \cdot \cos \left(\varphi-\gamma_{0}-\omega t\right)\right)
$$

where $\omega$ is rotation speed in rad/s and $t$ is the time.

\section{B. Model of Axis Misalignment}

The axis misalignment occurs when the stator and rotor are offset from each other in the axis direction, As shown in Fig. 5.

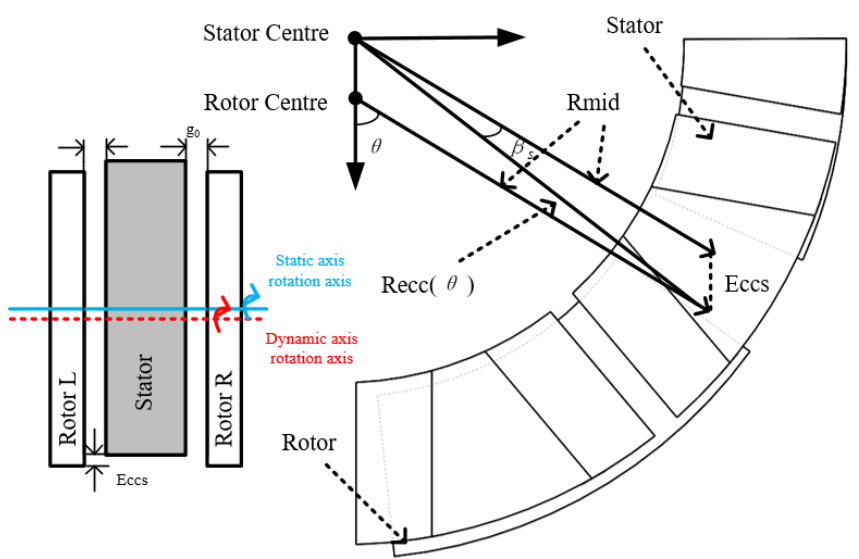

Fig. 5. Configuration of static/dynamic axis misalignment.

In order to reduce the complexity of the axis misalignment computations, the parts of poles overhanging the outer radius and the inner radius of the stator are ignored. The radius $\left(R_{e c c}\right)$ and its corresponding angle $\left(\beta_{s}\right)$ under static axis misalignment condition is deduced as:

$$
\begin{gathered}
R_{e c c}(\theta)=\sqrt{R_{m i d}^{2}+E_{c c s}^{2}-2 R_{\text {mid }} E_{c c s} \cos \left(\pi-\theta_{s}\right)} \\
\beta_{s}=\operatorname{acos}\left(\frac{R_{m i d}^{2}+R_{e c c}^{2}-E_{c c s}^{2}}{2 R_{m i d} R_{e c c}}\right)
\end{gathered}
$$

where $E_{c c s}$ is the maximum offset distance and $\theta_{s}$ is the location of the slot at angle $\theta$.

A similar process is done to deduce the radius equation of dynamic axis eccentricity, and the radius of dynamic axis eccentricity is:

$$
R_{e c c}(\theta)=\sqrt{R_{\text {mid }}^{2}+E_{c c s}^{2}-2 R_{\text {mid }} E_{c c s} \cos \left(\pi-\theta_{s}-\omega t\right)}
$$

\section{RESULTS AND DISCUSSION}

Since the prototype machine has two rotors and one stator, the flux linkage of the $k_{t h}$ tooth coil with $N_{c}$ turns is simply given by:

$$
\psi_{k}=N_{c}\left(\psi_{c, i, L k}+\psi_{c, i, R k}\right) / 2
$$

where $\psi_{c, i, L k}$ and $\psi_{c, i, R k}$ are the flux linkage caused by the two rotors (rotor left and rotor right) respectively.

The back-EMF per phase is calculated by considering all coils of the phase winding connected in parallel. The backEMF of phase $\mathrm{A}$ is given by:

$$
E_{A}=-\sum_{i}^{n_{s}} \frac{R_{a v e, i}\left(R_{o}-R_{i}\right)}{n_{s}} \frac{d \psi_{k}}{d t}
$$

The calculation route is descripted in Fig. 6. It should be noted that other manufacturing imperfections could be calculated by combining the provided equations of each independent case. For instance, the bearing faults and the shaft bending cases shown in Fig. 7 could be calculated by changing corresponding parameters in eq. (14) and eq. (15).

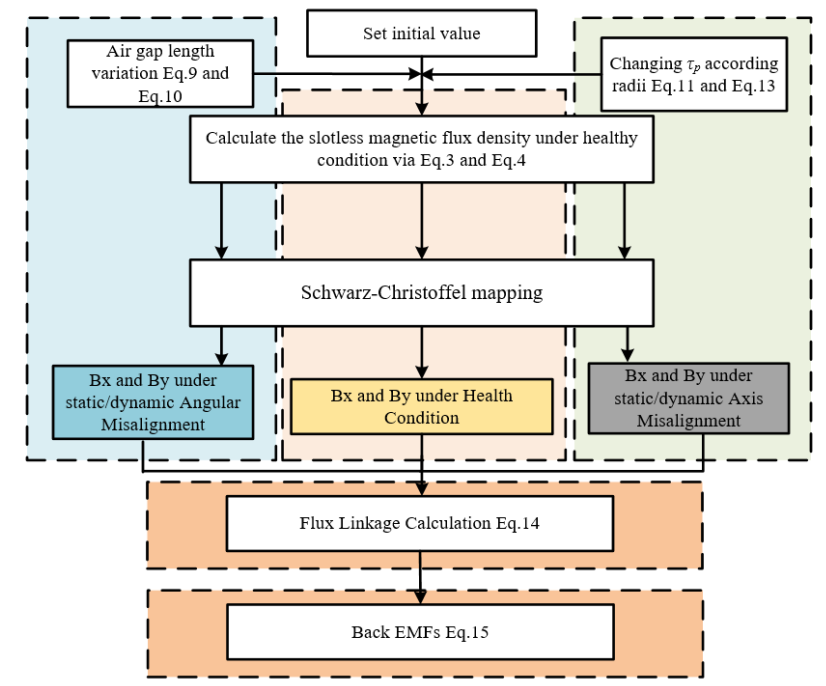

Fig. 6. Configuration of calculation processes.
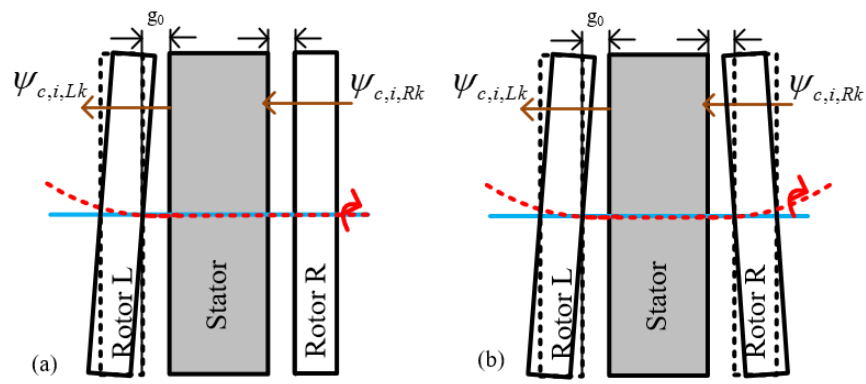

Fig. 7. Configuration of shaft bending cases. (a) Single shaft bending. (b) Double shaft bending.

\section{A. Results of Healthy Condition}

When AFPM machine operates under healthy condition, the air gap length is constant along the circumferential direction, the flux densities calculated by both FEM and the proposed method at the center of the airgap are shown in Fig.8. The calculated back EMFs are shown in Fig.9. It is indicated that the analytical results agree well with the FE results.

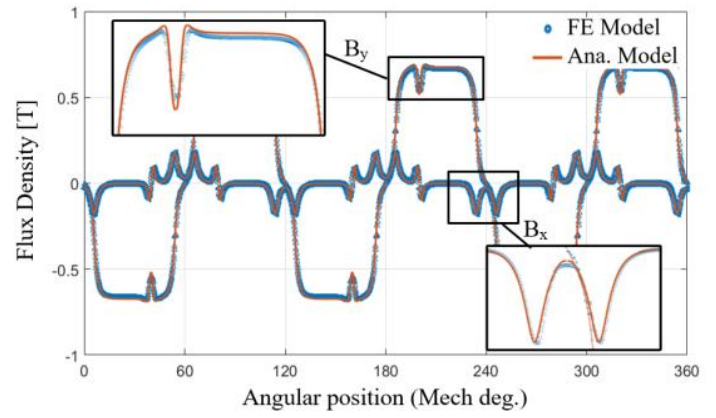

Fig. 8. Magnetic flux densities under healthy condition. 


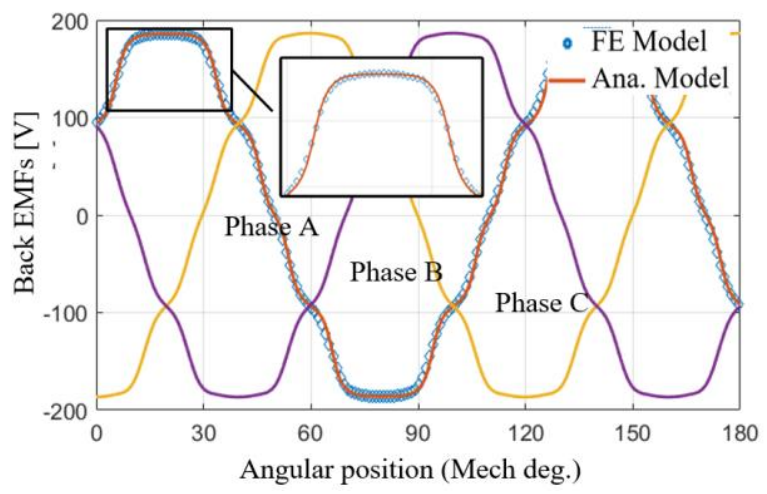

Fig. 9. Back EMFs under healthy condition.

\section{B. Results of Angular Misalignment}

$\mathrm{SEF}=40 \%$ (1.2mm deviation) is chosen to verify the effectiveness of the proposed method under angular misalignment condition. The reference point $\left(\gamma_{0}\right)$ is set as 0 degree. The axial and circumferential magnetic flux densities obtained by both FEM and the proposed method are shown in Fig. 10. Since the air gap length at different angle is different, the amplitude of flux density also changes with different angle. The calculated back EMFs of phase A by the proposed method are shown in Fig.11. It is shown that the back EMFs have a slight increment compared with healthy condition. It should be noted that the machine investigate in this paper is a double rotor AFPM machine with two air gaps, therefore, the flux density increase in one airgap, but decreases in the other airgap. As a result, the changes in the back EMF of the coils of one phase cancels each other, so that the back EMF results don't change much compared with healthy condition.

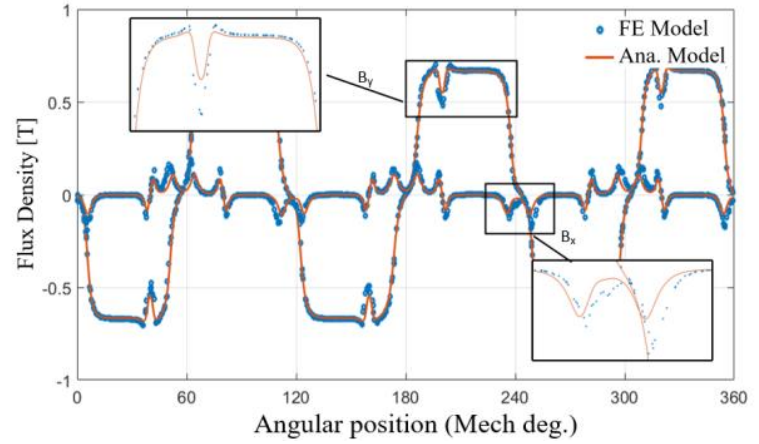

Fig. 10. Magnetic flux densities under angular misalignment condition.

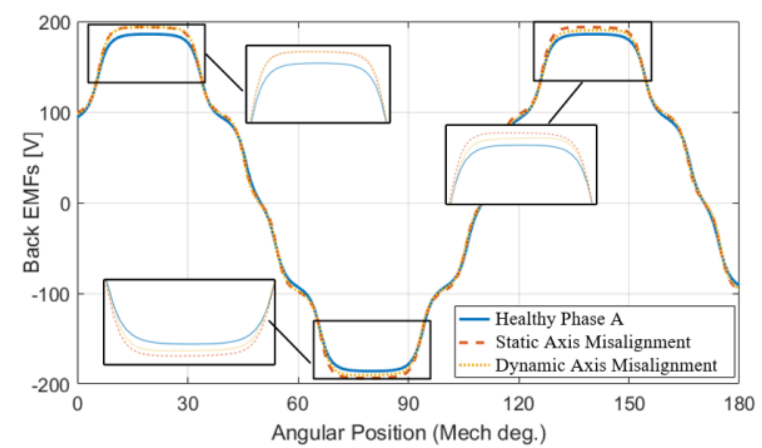

Fig. 11. Back EMFs of phase A under angular misalignment.

Fig.12 shows the FFT of the back EMF of phase A. It is shown that the third harmonics of the angular misalignment condition have only slight incensement compared with healthy condition. Therefore, the angular misalignment has small effect on the back EMFs.

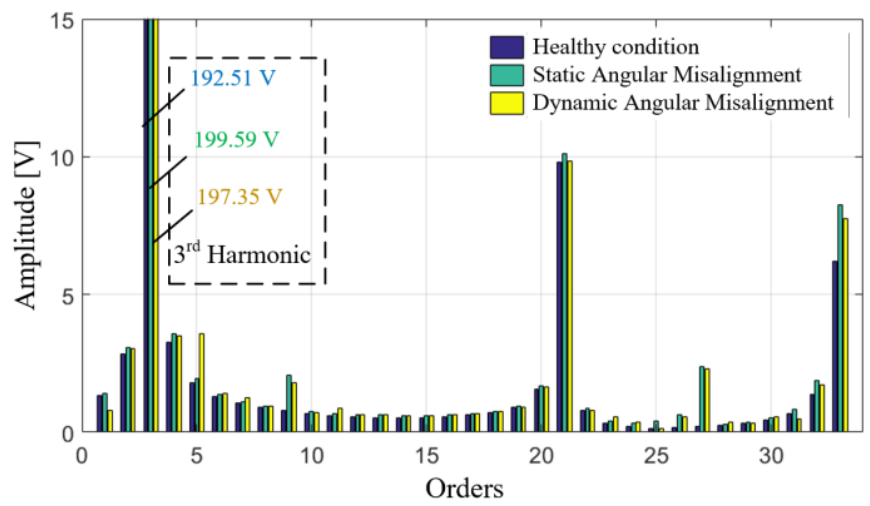

Fig. 12. The FFT of phase A under angular misalignment.

\section{Results of Axis Misalignment}

$E_{c c s}$ is set to $4 \mathrm{~mm}$ and the initial angle $(\theta)$ shown in Fig. 5 is set to 0 degree to verify the effectiveness of the proposed method. The magnetic flux densities and the back EMFs under axis misalignment condition are calculated by both FEM and the proposed method. The results are shown in Fig. 13 and Fig.14, respectively. It is shown that the results of the proposed method agree well with the FE model.

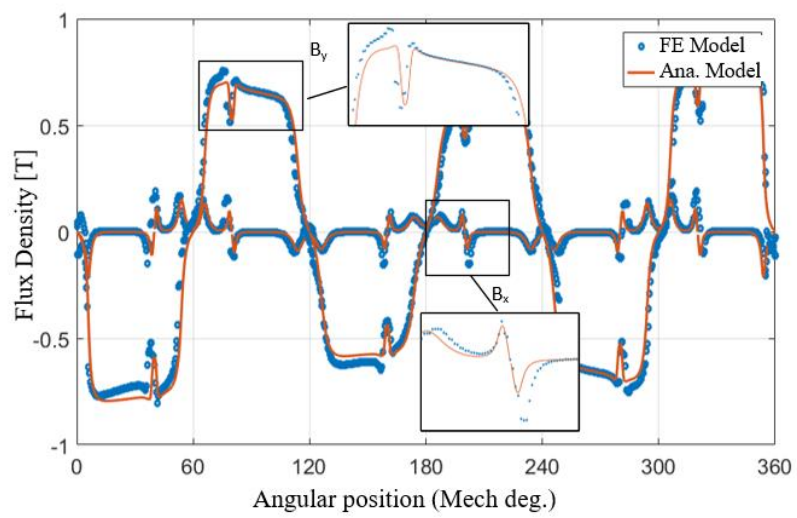

Fig. 13. Magnetic flux densities under axis misalignment condition.
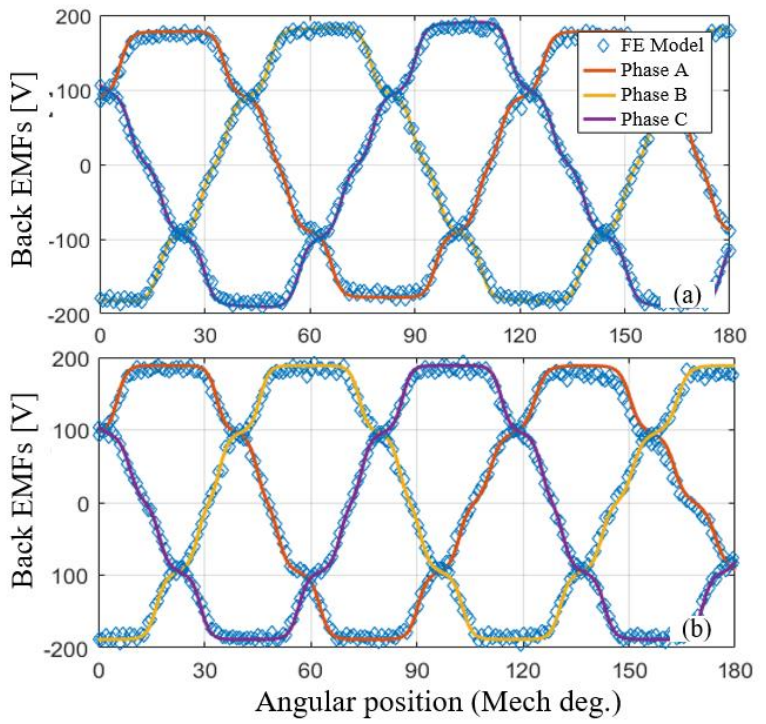

Fig. 14. Back EMFs under axis misalignment condition. (a) static axis misalignment, (b) dynamic axis misalignment. 
Fig. 15 shows the FFT analyze of the back EMF of healthy condition and axis misalignment condition. It is shown that the harmonics changes significantly with axis misalignments.

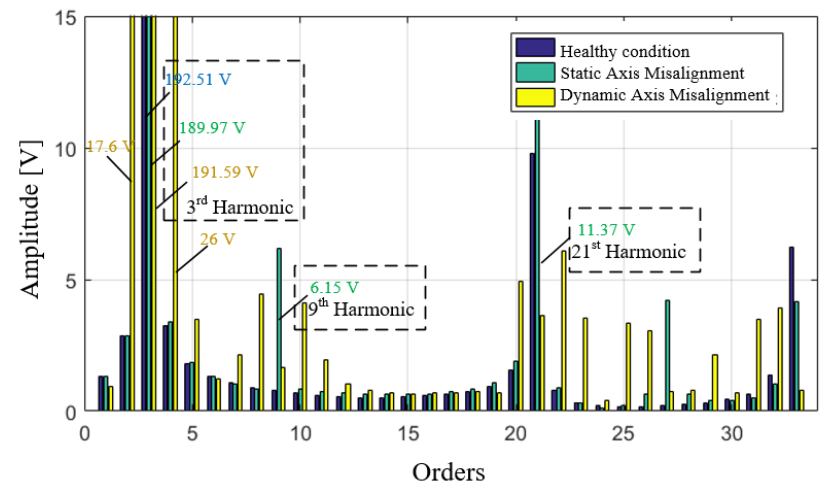

Fig. 15. The FFT of phase A under axis misalignment.

\section{Computation Time}

The CPU time of each model was compared in Table I. This comparison was done on a computer with Intel i7-4800 processor, 32 GB RAM and the 64 bit Windows 10 system.

The computation time of 3D FE model with one electrical cycle is provided in Table I. It shows that the analytical model is much faster than the FE model. When simulating the cases of manufacturing imperfections, the transient 3D full model is required. Therefore, the 3D full model takes much longer time than the proposed method.

TABLE I

CPU TIME COMPARISON

\begin{tabular}{cccc}
\hline \hline & \multicolumn{3}{c}{ Time } \\
\cline { 2 - 4 } Model & Analytical & 2D Multi-Slice & 3D Full \\
& Model & Model & Model \\
\hline Healthy Condition & 15 secs & 7 mins & - \\
Angular Misalignment & 76 secs & - & $27 \mathrm{hrs}$ \\
Axis Misalignment & 72 secs & - & $27 \mathrm{hrs}$ \\
\hline \hline
\end{tabular}

\section{EXPERIMENTAL RESULTS}

In order to verify the effectiveness of the proposed method, a dedicated test bench has been designed shown in Fig. 16. In this test bench, the motor prototype is coupled with an inductance machine by the belt system. the inductance machine is driven by an inverter. The motor prototype runs in generator mode.

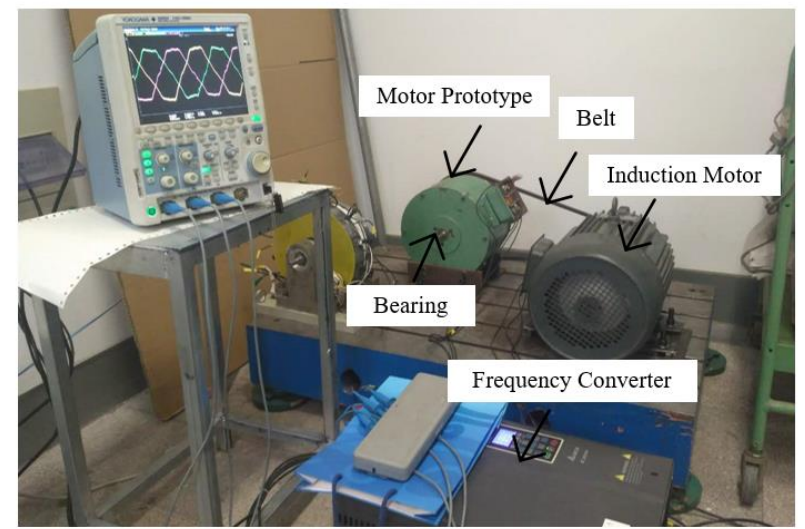

Fig. 16. Test bench view.

The measured back EMF under healthy condition at 640 $\mathrm{r} / \mathrm{min}$ is recorded. The results match well with the analytical results, as shown in Fig. 17.

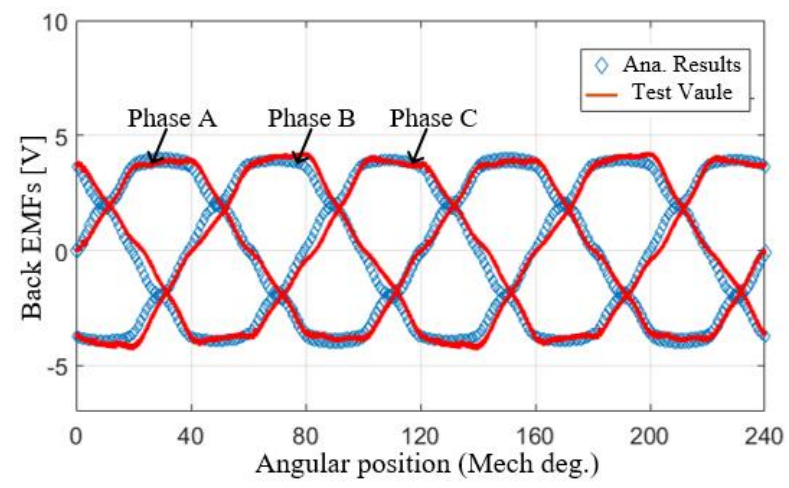

Fig. 17. The comparison between test and analytical results.

\section{CONCLUSION}

In this paper, an analytical approach is developed for modeling AFPMMs with various manufacturing imperfections. The model is verified by 3D FE model. The back EMFs of four types of misalignment have been investigated. It shows that angular misalignment does have much effect on the back EMFs. The axis misalignment changes the amplitude and phase of the back EMFs. In addition, the proposed method significantly reduces the computation time compared with FE model without losses much accuracy

\section{REFERENCES}

[1] M. Thiele and G. Heins, "Computationally Efficient Method for Identifying Manufacturing Induced Rotor and Stator Misalignment in Permanent Magnet Brushless Machines.," IEEE Trans. Ind. Appl., vol. PP, no. 99, pp. 1-1, 2016.

[2] A. Di Gerlando, G. M. Foglia, M. F. Iacchetti, and R. Perini, "Evaluation of Manufacturing Dissymmetry Effects in Axial Flux Permanent-Magnet Machines: Analysis Method Based on Field Functions," IEEE Trans. Magn., vol. 48, no. 6, pp. 1995-2008, Jun. 2012.

[3] E. Ajily, M. Ardebili, and K. Abbaszadeh, "Magnet Defect and Rotor Eccentricity Modeling in Axial-Flux Permanent-Magnet Machines via 3-D Field Reconstruction Method," IEEE Trans. Energy Convers., vol. PP, no. 99, pp. 1-10, 2015.

[4] Y. Huang, J. Zhu, Y. Guo, Z. Lin, and Q. Hu, "Design and Analysis of a High-Speed Claw Pole Motor With Soft Magnetic Composite Core," IEEE Trans. Magn., vol. 43, no. 6, pp. 2492-2494, Jun. 2007.

[5] Y. Huang, T. Zhou, J. Dong, H. Lin, H. Yang, and M. Cheng, "Magnetic Equivalent Circuit Modeling of Yokeless Axial Flux Permanent Magnet Machine With Segmented Armature," IEEE Trans. Magn., vol. 50, no. 11, pp. 1-4, Nov. 2014.

[6] A. Hemeida and P. Sergeant, "Analytical Modeling of Surface PMSM Using a Combined Solution of Maxwell's Equations and Magnetic Equivalent Circuit," IEEE Trans. Magn., vol. 50, no. 12, pp. 1-13, Dec. 2014.

[7] T. A. Driscoll and L. N. Trefethen, Schwarz-Christoffel mapping. Cambridge; New York: Cambridge University Press, 2002.

[8] D. Zarko, D. Ban, and T. A. Lipo, "Analytical Solution for Electromagnetic Torque in Surface Permanent-Magnet Motors Using Conformal Mapping," IEEE Trans. Magn., vol. 45, no. 7, pp. 29432954, Jul. 2009.

[9] A. Alipour and M. Moallem, "Analytical magnetic field analysis of axial flux permanent-magnet machines using Schwarz-Christoffel transformation," in Electric Machines Drives Conference (IEMDC), 2013 IEEE International, 2013, pp. 670-677.

[10] S. M. Mirimani, A. Vahedi, F. Marignetti, and R. Di Stefano, "An Online Method for Static Eccentricity Fault Detection in Axial Flux 
Machines," IEEE Trans. Ind. Electron., vol. 62, no. 3, pp. 1931-1942, Mar. 2015. 